\title{
Selective filling of carbon nanotubes with metals by selective washing
}

\author{
Qiang Fu, Gisela Weinberg, Dang-Sheng Su*
}

Fritz-Haber-Institut der MPG, Faradayweg 4-6, D-14195 Berlin, Germany

*Corresponding author. Fax: +49-30-8413 4401; E-mail: dangsheng@fhi-berlin.mpg.de

\begin{abstract}
A straightforward method is presented to produce catalyst particle-filled carbon nanotubes. Based on capillarity and surface tension, a simple strategy was developed to selectively fill carbon nanotubes with $\mathrm{Fe}, \mathrm{Co}$, and Ni species using a two-step method consisting of impregnation and selective washing. By chosing the impregnation and washing solution properly, metal particles are produced exclusively inside the nanotubes. STEM and SEM investigations confirm the location of these metal particles is only inside the carbon nanotubes.
\end{abstract}

Key Words: Carbon nanotube; Self-organization; Chemical treatment

\section{Introduction}

Concave character of inner carbon nanotubes (CNTs) with confined space offer the possibility to generate metal particles with enhanced structural and chemical stability. In contrast to frequently applied microand mesoporous oxides, CNT is a neutral support with high inertness to many chemical reactions. It is electrically and thermally conductive, allowing for application in electro- and heterogenous catalysis with high selectivity provided that the catalyst particles are uniform in size and structure. The key aspect to realize this application is to insert metal particles selectively into CNTs.

Ajayan and Iijima [1] reported the filling of CNTs with $\mathrm{Pb}$ by heating a mixture of $\mathrm{CNTs}$ and $\mathrm{Pb}$ above the melting point of $\mathrm{Pb}$. Later, a similar method has been applied to insert other metals, oxides, and inorganic salts into CNTs [2, 3]. Green and coworkers [4] developed another route for insertion by simultaneous opening and filling CNTs in aqueous solutions. Typically, CNTs are refluxed in concentrated nitric acids containing precursor salts, by which CNTs can be opened and filled by the precursors [5]. In addition, the so-called "incipient wetness impregnation", which is the most common method for catalyst preparation in industry, has been also demonstrated to be a simple filling method [6, 7].

All the above-mentioned filling processes make use of capillarity of CNTs. Liquid phases, which may be high-temperature molten materials, aqueous solutions, or organic solutions, can be sucked into the inner cavities of CNTs provided that the filling liquids have relatively low surface tension, below a cut-off value at $100-200 \mathrm{mN} / \mathrm{m}[8,9]$. The results have indicated that these procedures inevitably result in the deposition of a certain amount of materials onto external walls of the CNTs [5]. To achieve exclusive filling of CNTs, a simple strategy is suggested, illustrated in Fig.1. In step I, open CNTs are impregnated with excess of the precursor solutions under ultrasonic conditions to ensure that most of the tubes can be filled by the impregnating solution. In step II, a suitable washing medium is used to dissolve the solution sticking onto the external walls of CNTs but keep the solution in the internal cavity unchanged. The key point in this method is to choose a right washing medium.
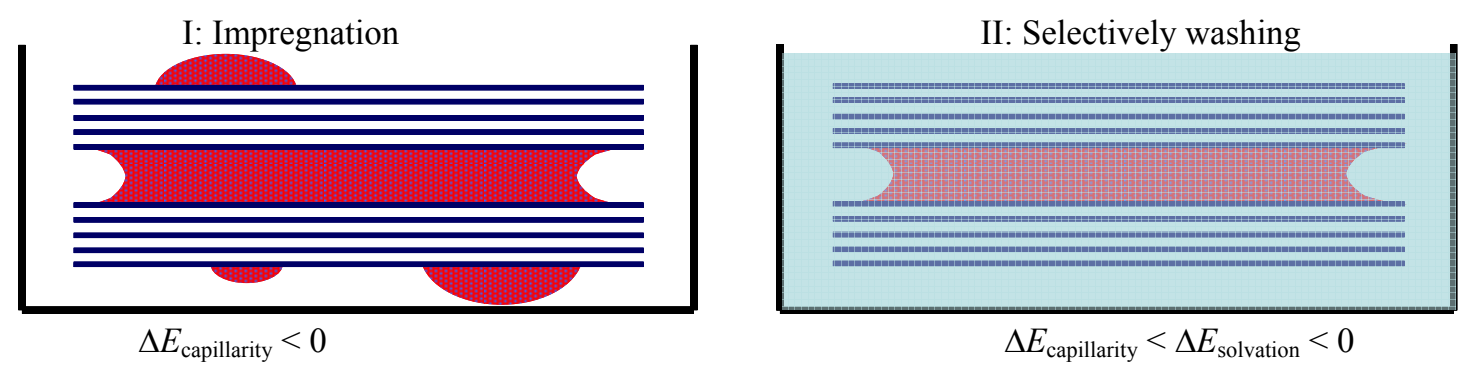

Fig.1 Schema of the selective filling of carbon nanotubes with metals: filling of CNTs via impregnation (I) and selective washing (II) 
Capillarity occurs if $\gamma_{S V}$, the surface tension at the solid-vapor interface for a dry tube is larger than $\gamma_{S L}$, the surface tension at the solid-liquid interface for the same tube during impregnation. The impregnation parameter, $I$, is defined as: $I=\gamma_{S V}-\gamma_{S L}$ [10]. According to Young's equation $\left(\gamma_{S V}-\gamma_{S L}=\gamma \cos \theta_{E}, \gamma\right.$ : surface tension of liquid and $\theta_{E}$ : equilibrium contact angle), the impregnation criterion can be written as: $I=\gamma_{S V}$ $\gamma_{S L}=\gamma \cos \theta_{E}>0$. Wetting, i.e. $\theta_{E}<90^{\circ}$, is necessary for capillarity action in CNTs [2,8]. The energy gain for the capillary filling of a CNT (with length of $l$ and radius of inner cavity of $R$ ) by an impregnating liquid (step I in Fig.1) is as follows:

$$
E_{\text {capillarity }}=-2 R l \cos \theta_{E} \text {. }
$$

The dissolution of the impregnating solution into the washing medium (step II in Fig.1) is driven by the solvation energy. The process of transforming state 1 (isolated impregnating phase and washing phase) to state 2 (dissolution of impregnating phase in washing phase) is associated with a change in free energy:

$$
E_{\text {solvation }}=G_{2}-G_{1}=\Delta H-T \Delta \mathrm{S},(2)
$$

$G_{1}$ and $G_{2}$ are the energies at the states 1 and 2, respectively. The energy change is due to the enthalpic and entropic changes during solvation [11].

From the thermodynamic point of view, the dissolution of the impregnating solution sticking to external walls of CNTs into the washing medium necessitates the condition of $\Delta E_{\text {solvation }}<0$. On the other hand, $\Delta E_{\text {capillarity }}<\Delta E_{\text {solvation }}$ is required to ensure that the solution is stable in the internal cavity of CNTs, otherwise, it may dissolve into the outside washing medium. In summary, to achieve the selective washing shown in Fig. 1, the following inequality is needed: $\Delta E_{\text {capillarity }}<\Delta_{\text {Esolvation }}<0$

The above inequality suggests that the impregnating solution and washing medium should be carefully chosen such that the impregnating solution should present limited solubility in the washing medium. Green and coworkers found that washing the CNTs which were filled by aqueous solutions of inorganic compounds in water removed almost all materials from both inside and outside of the tubes [3,5]. However, organic solvents, such as chloroform and benzene, are completely immiscible with CNTs impregnated with aqueous solutions and will not remove any excess precursor solutions.

In this study, the filling strategy is exemplified by showing the selective filling of CNTs with $\mathrm{Fe}, \mathrm{Co}$, and Ni. $0.18 \mathrm{~mol} / \mathrm{L} \mathrm{Fe}\left(\mathrm{NO}_{3}\right)_{3}, 0.09 \mathrm{~mol} / \mathrm{L} \mathrm{Co}\left(\mathrm{NO}_{3}\right)_{2}$, and $0.09 \mathrm{~mol} / \mathrm{L} \mathrm{Ni}\left(\mathrm{NO}_{3}\right)_{2}$ were used as the impregnating solutions. A mixture of dichloroform and 1-propanol with volume ratio of 4:1 was chosen as the washing agent. Such a mixture enables miscibility between the impregnation solutions and the washing agent. Furthermore, a limited solubility of the impregnating solutions in the mixture assures that the solution sucked into the internal cavity of CNTs will not be washed away.

\section{Experimental}

Open CNTs with inner diameter in the range of 50-150 nm were acquired from Applied Science Ltd. (Ohio, USA). The tubes were free of impurities and amorphous carbon. The as-received CNTs were subjected to treatment with boiling nitric acid for $3 \mathrm{~h}$ to make the tubes hydrophilic. They were then impregnated with excess of $0.18 \mathrm{~mol} / \mathrm{L} \mathrm{Fe}\left(\mathrm{NO}_{3}\right)_{3}$ solution. The impregnation was facilitated by the ultrasonic treatment which helps to fill most tubes by the impregnating solution. The impregnated CNTs were subsequently washed by the mixture of dichloroform and 1-propanol. The suspension was filtered and dried at $60{ }^{\circ} \mathrm{C}$ overnight. Calcination of the samples was performed at $350{ }^{\circ} \mathrm{C}$ under flowing $\mathrm{He}$ for 2 $\mathrm{h}$ followed by reduction with flowing $\mathrm{H}_{2}$ for $2 \mathrm{~h}$ at $400{ }^{\circ} \mathrm{C}$. This procedure was applied to CNTs filled with Co and Ni. A Hitachi S-4800 scanning electron microscope (SEM) was used for the characterization.

\section{Results and discussion}

All the three prepared samples were investigated using the Hitachi SEM operating at transmission mode at $30 \mathrm{kV}$. The typical S(T)EM images were shown in the left column of Fig. 2. Image contrast shows that iron, nickel, and cobalt particles are formed on/in CNTs. To localize whether the metal is inside or outside CNTs, the accelerating energy of primary electrons was reduced in the SEM to $1.5 \mathrm{kV}$. It is known that in case of low energy primary electron beams, SEM gives only surface sensitive image.

The surface sensitive SEM investigations demonstrate that the outer surfaces of the CNTs are very smooth and almost free of metal particle decoration (right column of Fig.2), indicating that metal particles are almost exclusively filled inside CNTs. This suggests that selective impregnation of CNTs with iron, nickel, and cobalt has been successfully achieved by the simple selective washing method. 
It should be mentioned that the micrographs in Fig. 2 reveal that the prepared iron and nickel particles have very similar, but also narrow particle size distribution. This is not the case for cobalt particles. It is obvious that the chosen above- described experimental condition guarantees only filling the metal particles into the CNTs, which is the purpose of the current article. By changing the concentration of cobalt salts in the solvent, the size of cobalt particles can be controlled.
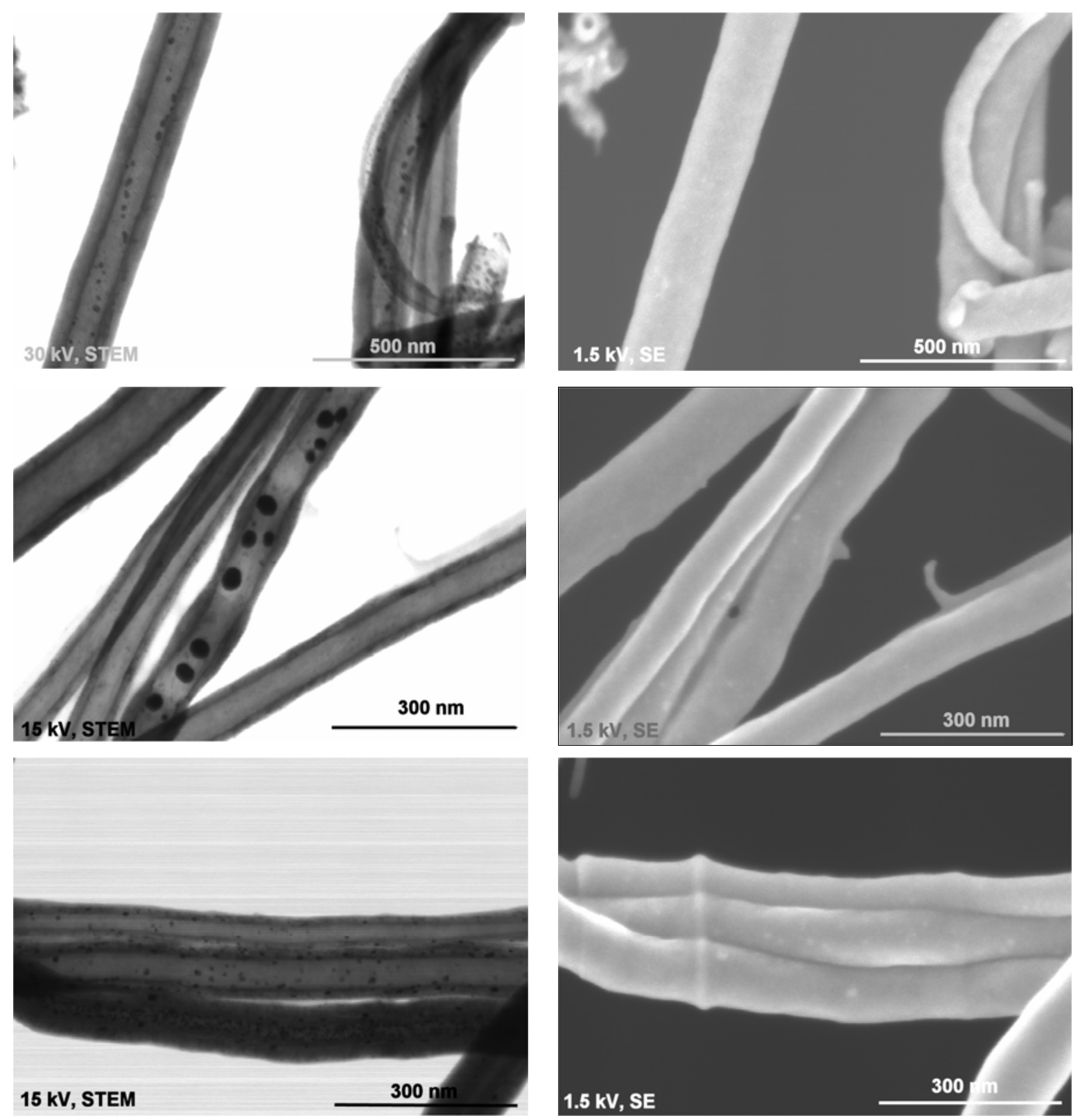

Fig.2 Scanning transmission electron micrographs (left column) and SEM (right column) images of Fe-filled (top), Co-filled (middle) and Ni-filled (bottom) CNTs

\section{Conclusions}

In summary, a simple strategy to fill CNTs with metal particles via selective washing using a dichloroform/propanol mixture is suggested. Iron, cobalt, and nickel particles have been selectively filled inside the CNTs by this method, which depends on the solvation conditions between the impregnating solution and washing medium. Since the filling strategy makes use of capillarity effects, this simple method can be applied to selectively fill other tubular, mesoporous, and microporous materials.

\section{Acknowledgments}

The study was supported by the EU NEST framework project ELCAT. D.S.S thanks Prof. R. Schlögl for critical discussions. 


\section{References}

[1] Ajayan P M, Iijima S. Capillarity-induced filling of carbon nanotubes. Nature, 1993, 361 (6410): 333-334.

[2] Ugarte D, Chatelain A, de Heer W A. Nanocapillarity and chemistry in carbon nanotubes. Science, 1996, 274 (5294): 1897-1899.

[3] Thamavaranukup N, Höppe H A, Ruiz-Gonzalez L, et al. Single- walled carbon nanotubes filled with MOH $(\mathrm{M}=\mathrm{K}, \mathrm{Cs})$ and then washed and refilled with clusters and molecules. Chem Commun, 2004(15): 1686-1687.

[4] Tsang S C, Chen Y K,. Harris P J F,et al. A simple chemical method of opening and filling carbon nanotubes. Nature, 1994, 372 (6502): 159-162.

[5] Lago R M, Tsang S C, Lu K L, et al. Filling carbon nanotubes with small palladium metal crystallites: the effect of surface acid groups. Chem Commun, 1995 (13): 1355-1356.

[6] Pham-Huu C, Keller N, Estournes C, et al. Synthesis of $\mathrm{CoFe} 2 \mathrm{O} 4$ nanowire in carbon nanotubes. A new use of the confinement effect.Chem Commun, 2002(17): 1882-1883.

[7] Tessonnier J P, Pesant L, Ehret G, et al. Pd nanoparticles introduced inside multi-walled carbon nanotubes for selective hydrogenation of cinnamaldehyde int hydrocinnamaldehyde. Appl Catal, A 2005,288 (1-2): 203-210.

[8] Dujardin E, Ebbesen T W, Hiura H, et al. Capillarity and Wetting of Carbon Nanotubes. Science, 1994, 265 (5180): 1850-1852.

[9] Ebbesen T W. Wetting, filling and decorating carbon nanotubes. Phys Chem Solids, 1996,57 (6-8): 951-955.

[10] De Gennes P G, Brochard-Wyart F, Quere D.Capillarity and Wetting Phenomena: Drops, Bubbles, Pearls, Waves. Springer, 2003: 51-52.

[11] Chandler D. Interfaces and the driving force of hydrophobic assembly. Nature, 2005, 437 (7059): 640-647. 